\title{
A Focus on Medication Error
}

\section{Prashansa Agrawal ${ }^{*}$}

Department of Chemistry, Case Western Reserve University, Cleveland, Ohio, 44106, USA

*Corresponding author: Prashansa Agrawal, Department of Chemistry, Case Western Reserve University, Cleveland, Ohio, 44106, USA, Tel: +1 216-368-2404; Email: prashansa.agrawal@case.edu

Received date: July 13, 2015; Accepted date: July 13, 2015; Published date: July 16, 2015

Copyright: (c) 2015 Agrawal P. This is an open-access article distributed under the terms of the Creative Commons Attribution License, which permits unrestricted use, distribution, and reproduction in any medium, provided the original author and source are credited.

\section{Editorial}

A medication error is any avoidable adverse incident that happens due to negligence or incorrect administration of the medication to the patients that lead to an erroneous or imperfect diagnosis of a disease, injury, syndrome, behaviour, infection or other illness. The cause of medication error can be associated to healthcare professionals' careless practice, outdated health care products, improper follow-ups of the procedures and systems. There are various factors involved that promote such events to happen, for example, an error in dosage or route of administration, incorrect prescription, problem in reading handwritten prescription, failure to administer the correct formulation for a particular disease, use of expired drugs, incorrect timing for administration of the drug, lack of awareness of adverse effects of certain drug combinations, shortage of information about a patient's drug allergies or sensitivities, problem with product labelling, packaging, compounding, dispensing, distribution, monitoring, administration and nomenclature, that is, confusion about different drugs with similar names.

The National Coordinating Council for Medication Error Reporting and Prevention (NCC MERP) has organized medication errors into four major groups [1]: (a) No error- events that have the ability to cause error. (b) Error but no harm- (i) an error happened but did not reach the patient. (ii) An error occurred and reached the patient but did not cause any harm to the patient. (iii) An error happened and reached the patient but required monitoring to confirm that it did not harm the patient and/or required intrusion to prevent harm. (c) Error and harm- (i) an error occurred that may have contributed to temporary harm to the patient and required intervention. (ii) An error happened that may have contributed to temporary harm the patient but also required early or long-term hospitalization. (iii) An error occurred that may have resulted into permanent harming the patient and (iv) an error occurred that need mandatory intervention to sustain the life of the patient. (d) Error and death- An error occurred that may have resulted in the patient's death.

Universally, medication errors affect one in 10 patients. A report by Institute of Medicine in year 2000, estimated that medication errors have resulted in 44,000-98,000 preventable deaths and over 1,000,000 injuries each year in United States hospitals [2-4]. In United Kingdom, approximately 850,000 medication errors occur every year, costing over $£ 2$ billion [5]. In 2001, a report on seven medical centers of Department of Veterans Affairs, in Journal of the American Medical Association, estimated that approximately every 10,000 patients admitted to the hospitals, out of which one die due to medical error [6]. Worldwide it is estimated that 142,000 people died in 2013 from adverse effects of medical treatment in comparison to 94,000 in 1990 [7]. Approximately 180,000 people die each year partially as a result of iatrogenic injury [8]. One out of every five Americans (22\%) are reported to have some kind of medication error experience [9].
The medication errors are amongst the most common medical mistakes, hurting $\sim 1.5$ million people every year [10]. According to the study, 400,000 avertible drug-related injuries occur each year in hospitals, 800,000 in long-term care surroundings, and $~ 530,000$ among medicare patients in outpatient clinics. In 2000 alone, the extra medical expenses incurred by avoidable drug associated injuries were $\sim 887$ million. Medication errors may be due to the failure of a medication plan to be completed as anticipated or the use of an incorrect medication plan to achieve a goal. Some examples of medication errors are: Celebrex (anti-inflammatory) is mistaken for Celexa (antidepressant). Zyrtec (antihistamine) is mistaken for Zyprexa (antipsychotic). $0.5 \mathrm{mg}$ of Xanax is mistaken for $5 \mathrm{mg}$ of Xanax. An MD's verbal order for Toradol $15 \mathrm{mg}$ is mistaken for $50 \mathrm{mg}$. Insulin $5 \mathrm{u}$ is mistaken for 50 units. Amoxicillin is ordered for a patient with a penicillin allergy.

Documentation of medication error is crucial for avoiding its reoccurrence in future. In 1999 Institute of Medicine (IOM) report, To Err Is Human describes two primary purposes of reporting program of medication error [2]: (i) providers should hold accountability for performance and patient safety. (ii) They need to provide information that leads to new knowledge and improved patient safety. In addition, it is essential to use clear, consistent terminology when reporting these events. Examples of safer systems include: Computerized medication record systems, designing and utilizing safer systems reduces the number and severity of events. People make mistakes, but good systems design and continuous improvements utilizing the information obtained from error analyses have been shown to resolve errors.

The goal of The Joint Commission National Patient Safety is to accurately and completely reconcile medications across the continuum of care. Medication error can be prevented by following at least these 7 basic rights: Right patient, right medication, right dose, right route, right time, right form and right documentation. Care should be taken to complete the admission database of patients by obtaining a good patient medication history so that adverse drug reactions can be avoided, accurate prescription of drugs and dosages, correct over-thecounter drugs and dosages, herbal/alternative products, rewrite orders using a medication order summary form or the copy of Medication Administering Record (MAR) should be mandatory.

In conclusion, it is pivotal to make safety top priority and move from a culture of blame to one of safety, as to err is human. Improvement of the system, utilization of the technology as it becomes available, involvement of the patients to the fullest extent, and iterative refinement is essential for safer medication. Care should be taken to ensure that the medication is given in a safe and secure manner, by checking 3 things, MAR, doctors' orders and pharmacy label. Precaution should be taken to ascertain the right person, right medication and right route. Checking the generic with the brand is also very important for surety of the right medication. Safe disposition 
of the expired medicines is crucial. Consumers who self-medicate should meet the nurse to ensure that they are taking the medication in a safe manner. At last, all the above-mentioned factors are vital for reconciliation of medication errors, which is essential for building a safer health system.

\section{References:}

1. NCC MERP, NCC MERP Index for Categorizing Medication Errors.

2. Kohn LT, Janet M. Corrigan, Molla S. Donaldson (2000) To Err Is Human: Building a Safer Health System. Washington, DC: National Academies Press.

3. Charatan F (2000) Clinton acts to reduce medical mistakes. BMJ 320: 597.

4. Weingart SN, Wilson RM, Gibberd RW, Harrison B (2000) Epidemiology of medical error. BMJ 320 (7237): 774-777.
5. Ker K, Edwards PJ, Felix LM, Blackhall K, Roberts I (2010) Caffeine for the prevention of injuries and errors in shift workers. Cochrane Database of Systematic Reviews 5.

6. Hayward RA, Hofer TP (2001) Estimating hospital deaths due to medical errors: preventability is in the eye of the reviewer. JAMA 286: 415-420.

7. GBD 2013 Mortality and Causes of Death, Collaborators (2014) Global, regional, and national age-sex specific all-cause and cause-specific mortality for 240 causes of death, 1990-2013: a systematic analysis for the Global Burden of Disease Study 2013. Lancet 385: 117-171.

8. Leape LL (1994) Error in medicine. JAMA 272: 1851-1857.

9. Annual Report (2002) The Commonwealth Fund.

10. Medication Errors Injure 1.5 Million People and Cost Billions of Dollars Annually (2006) The National Academy of Science. 